CrossMark <click for updates

Cite this: New J. Chem., 2015, 39, 9030

DOI: 10.1039/c5nj90050f

www.rsc.org/njc

\title{
Correction: Conformational features of 4-(N)- squalenoyl-gemcitabine in solution: a combined NMR and molecular dynamics investigation
}

\author{
Maurizio Ceruti, ${ }^{a}{ }^{\text {Flavio Rocco, }}{ }^{a}$ Maria Enrica Di Pietro, ${ }^{b}$ Elena Tocci $^{\mathrm{c}}$ and \\ Giuseppina De Luca*b
}

Correction for 'Conformational features of 4-(N)-squalenoyl-gemcitabine in solution: a combined NMR and molecular dynamics investigation' by Ceruti Maurizio et al., New J. Chem., 2015, 39, 3484-3496.

The authors regret the exchange between the first and last names of the authors in the original manuscript. As it is important to correctly identify each author, the authors wish to inform readers of the correct order of their names. The correct order of name and surname of the authors for this paper is as shown above.

The Royal Society of Chemistry apologises for these errors and any consequent inconvenience to authors and readers.

\footnotetext{
${ }^{a}$ Dipartimento di Scienza e Tecnologia del Farmaco, Università degli Studi di Torino, Via P. Giuria 9, 10125 Torino, Italy. E-mail: maurizio.ceruti@unito.it

${ }^{b}$ Dipartimento di Chimica e Tecnologie Chimiche, Università della Calabria, Via P. Bucci, cubo 14C, 87036 Rende (CS), Italy. E-mail: giuseppina.deluca@unical.it

${ }^{c}$ Institute on Membrane Technology (ITM-CNR), Via P. Bucci 17/C, 87036 Rende (CS), Italy
} 